\title{
Opportunistic Spectrum Access with Dynamic Users: Directional Graphical Game and Stochastic Learning
}

\author{
Yuli Zhang ${ }^{1,2}$, Yuhua $\mathrm{Xu}^{1,2}$ and Qihui $\mathrm{Wu}^{3}$ \\ ${ }^{1}$ College of Communication Engineering, PLA University of Science and Technology, Nanjing, China, 210007 \\ ${ }^{2}$ Science and Technology on Communication Networks Laboratory, Shijiazhuang, 050002, China \\ [e-mail: yulipkueecs08@126.com, yuhuaenator@gmail.com.] \\ ${ }^{3}$ College of Electronic and Information Engineering, Nanjing University of Aeronautics and Astronautics \\ Nanjing, China, 210007 \\ [e-mail: wuqihui2014@sina.com] \\ *Corresponding author: Yuhua Xu
}

Received January 11, 2017; revised June 7, 2017; accepted July 7, 2017;

published December 31, 2017

\begin{abstract}
This paper investigates the channel selection problem with dynamic users and the asymmetric interference relation in distributed opportunistic spectrum access systems. Since users transmitting data are based on their traffic demands, they dynamically compete for the channel occupation. Moreover, the heterogeneous interference range leads to asymmetric interference relation. The dynamic users and asymmetric interference relation bring about new challenges such as dynamic random systems and poor fairness. In this article, we will focus on maximizing the tradeoff between the achievable utility and access cost of each user, formulate the channel selection problem as a directional graphical game and prove it as an exact potential game presenting at least one pure Nash equilibrium point. We show that the best NE point maximizes both the personal and system utility, and employ the stochastic learning approach algorithm for achieving the best NE point. Simulation results show that the algorithm converges, presents near-optimal performance and good fairness, and the directional graphical model improves the systems throughput performance in different asymmetric level systems.
\end{abstract}

Keywords: Opportunistic spectrum access, directional graphical game, dynamic users, asymmetric interference relationship, stochastic learning approach. 


\section{Introduction}

$\mathbf{O}_{\text {pportunistic spectrum access (OSA) has recently drawn great attention as a promising }}$ technique for solving the spectrum shortage $[1,2]$. Among the reproted works, the common preassumptions for users and interference are as follows. Users are transmitting all the time, and the interference between two users is symmetric. However, in practical applications, whether users will transmit depend on their traffic demands, which means they are switched between active and inactive. Besides, due to the fact that different users have different interference ranges, they may not affect each other at the same time. Thus, the interference relations among users are not always symmetric, As a result, the channel selection problem in OSA systems with dynamic users and asymmetric interference relation is more practical. However, it has drawn quite little attention.

This paper focuses on the channel selection problem of dynamic users and asymmetric interference relation in distributed OSA systems. Compared with the static users, dynamic users exhibit some differences. For example, in the dynamic user situation, when all users keep their channel selections unchanged, the interference level and the throughput rewards received in each slot may be random and uncertain. In contrast, in the static situation, these feedbacks are static and determined. In addition, dynamic users lead to poor fairness. When sharing the same channel, the users with high active probabilities seize the transmitting opportunity from the low active probabilities ones. Thus, the ratio of their throughput does not match with their active probabilities, which is unfair and should be avoided. Moreover, the asymmetric interference relation between the users yields a more intractable channel selection by unilateral influence. To the best of our knowledge, no work has been reported the analysis of the asymmetric interference relations using graphical game solutions, while only few simulation work have been presented in [3,4]. Therefore, solving the channel selection problem between the presence of dynamic users and asymmetric interference relation is a challenging task.

Here, we assume that, at each time slot, each user is randomly active or inactive. In addition, we consider that the interference relation between users may be asymmetric, and then model it as a directional graph. We focus on the trade-off between the achievable utility and the access cost of each user. The channel selection problem is formulated as a directional graphical game. Moreover, the proposed system model employs the stochastic learning approach (SLA) [5]. Simulation results show that the SLA algorithm achieves near optimal performance and the directional graphical model improves the system's throughput.

The contributions of this paper are as follows.

- A practical situation, where the interference relationship is asymmetric and the users randomly transmit the data based on their dynamical traffic demands is considered in this paper.

- The channel selection problem of the dynamic users and asymmetric interference relation is modeled as a directional graphical game. The proposed game is proved an exact potential game with at least one pure Nash equilibrium point.

- The stochastic learning algorithm is employed for the proposed game. Besides, while the access fails, the traditional positive access reward and users may also update the strategies with the negative access cost. 
- The simulation results show that the SLA algorithm achieves a near-optimal throughput performance with good fairness. The directional graphical model improves the system's throughput compared with non-directional models.

The rest of this paper is organized as follows. Section II presents related works. Next, Section III introduces the system model and formulate the problem. Then, we propose the directional graphical game in Sec. IV, and present its properties and modify the stochastic learning solution of the system model. Section $\mathrm{V}$ provides the simulation and discussion of the results, and Finally, Sec. VI concludes the paper.

\section{Related Works}

Over the past decades, game theory has been widely applied in solving the problem of distributed channel selection of OSA systems. However, most of the reported game models are non-spatial, which ignore the spatial relation among users. To overcome this issue, the graphical games have been recently proposed [3, 4, 6-9]. In [3], a graphical game formulation for distributed channel selection is introduced. The same problem is studied using the local minority game [6]. Authors in [8] realized a global optimization through the local altruistic game and local congestion game. They extended the graphical games to the partially overlapping channels situations in [9] and investigated the interference mitigation game in [4]. However, similar with other literatures, these graphical game models assume static users and symmetric interference relation.

Recently, the channel selection problem with dynamic users has regained attention. Authors in [10] studied the users' dynamic characteristics in distributed OSA systems. They focused on the interference mitigation while we studied the tradeoff between the achievable utility and the access cost. However, minimizing the interference level is not sufficient since the throughput directly reflects the system performance while the interference does not. Besides, they considered a symmetric interference relation, while this paper studies an asymmetric one, which is more common and practical. The most related work to the proposed problem in this paper was reported in [11], where the channel selection problem with spatial reuse dynamic users was investigated. However, they studied the problem considering users with fixed locations and user mobility, while the present study considers the asymmetric interference relation.

A research related of asymmetric interference is published recently. Authors studied the antenna selection and power allocation techniques to further improve the performance of topology management in asymmetric interference networks in [24]. Another recent work [25] is about the interference align and they proposed a spectrum-efficient topology management scheme to interference align in asymmetric networks. Compared with the existing asymmetric interference works, we focused on the channel selection problem in opportunistic spectrum access and solved it in a game-theoretic approach.

Opportunistic spectrum access has been recently applied to many problems. For instance, the Quality of Experience (QoE) of the users with less information exchange [17], and the anti-jamming problem through a cooperative spectrum access mechanism [18]. Besides, the network selection and the channel access may be combined to share the spectrum in 5G [19]. The spectrum sensing and access may be joint to provide a better throughput by a more accurate sensing result [20-21]. Also, the OSA is applied in the energy issues and some interesting problems are modeled as in it, such as energy harvesting and smart grids [22-23]. 
The difference between the present study and previous works may be categorized as follows: (i) We consider a problem with dynamic users instead of static users. (ii) An asymmetric interference relation between the users is assumed and the channel selection game is formulated as a directional graphical game. (iii) We make a tradeoff between the achievable utility and access cost.

\section{System Model and Problem Formulation}

\subsection{System Model}

Consider a distributed OSA system with multiple users and channels, where the set of users and the set of channels are denoted by $\mathcal{N}=\{1,2 \ldots N\}$ and $\mathcal{M}=\{1,2 \ldots M\}$. We ignore the situation that users may experience different channel conditions and assume that each channel offers the same transmitting rate for every user [12]. Denote the transmitting rate of the channel $m$ as $R m, m \in M$. Similar assumption may be found in IEEE 802 standards.

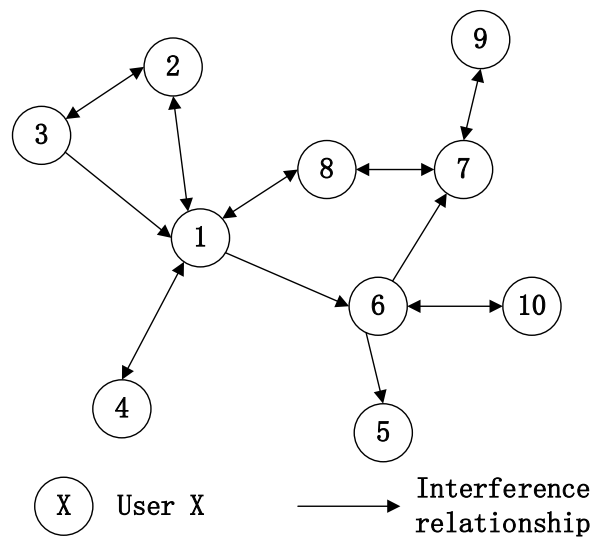

Fig. 1. An example of directional graphic interference model.

In practical communication systems, the users considerably affect some of the adjacent users. We use a directional graphical model to describe the users' local interference relation, and define the interference relation between user $n$ and user $m$ as $\delta(m, n)$ as

$$
\delta(m, n)=\left\{\begin{array}{l}
1, \text { user } m \text { affects user } n \\
0 \text {,user } m \text { doesnotaffect user } n
\end{array}\right.
$$

Furthermore, the situation of asymmetric interference relation is considered, where user $n$ affects user $m$ but not in the opposite way. The heterogeneous interference range represents the main reason leading to the asymmetric interference relation. We use an example to show the directional graphic model of interference relation in Fig. 1. The directions of arrows describe the interference relation between two users. The neighbor set of user $n, J_{n}$, is defined as

$$
J_{n}=\{m: \delta(m, n)=1, m \in M / n\}
$$

as the user set affecting the user $n$.

In many other related literatures, users are transmitting data all the time, while in reality, users transmitting the data are based on their traffic demand. Here, we assume that users are active to content for the channels with probability. The active probability for contending is defined as $\theta_{i}, 0<\theta_{i}<1, i \leq N$. Besides of the users' dynamic participation, we assume the following other practical characteristics of the OSA systems. (i) No centralized controller 
exists and the users make decisions, in a distributive and autonomous manner. (ii) No information exchange occurs between the users, while the users do not have any information about the others. For each user, the only helpful information is the access history.

The system transmitting structure is slotted, where for the active users, at the beginning of the slot, they only select one channel to access. If two neighbors both select the same channel for one slot, they collide and both fail in this slot. However, for two non-neighbor users that are far from each other, they may successfully transmit the data on the same channel and the same time slot. At the end of the slot, users update their channel selection strategies.

Remark 1: Note that here, the interference model of the users is a directional graph, which describes a more practical system than the non-directional. However, the directional graphical interference model is not well studied in the existing works.

\subsection{Problem Formulation}

The channel selection of user $n$ is defined as $a_{n} \in M$. The utility of user $n$ receiving from one slot is expressed as

$$
r_{n}(j)=\left\{\begin{array}{l}
R_{a_{n}}-c \text {, user } n \text { successfully transmits the data } \\
-c \text {, user } n \text { fails to transmit } \\
0, \text { user } n \text { is inactive }
\end{array}\right.
$$

where $c$ is the normalized access cost. The reason to consider the tradeoff between the throughput and access cost is as follows. (i) The contention behavior cost energy and time, and (ii) with the help of cost, it is easy to distinguish the inactive and fail situations. Users may also learn about the channel information from the fail experience.

Based on the description in the system model, user $n$ accesses the channel successfully only when user $n$ is active and its neighbors with same channel selection are inactive. Consider the active probabilities of user $n$ and its neighbors $J_{n}$, the expected utility of user $n$ is as follows,

$$
u_{n}\left(a_{1}, a_{2}, \ldots, a_{N}\right)=R_{a_{n}} \theta_{n} \prod_{i \in \mathbf{J}_{n}, a_{i}=a_{n}}\left(1-\theta_{i}\right)-\theta_{n} c,
$$

where $R_{a_{n}} \theta_{n} \prod_{i \in \mathbf{J}_{n}, a_{i}=a_{n}}\left(1-\theta_{i}\right)$ in Eq. (4) is the throughput received from the channel $a_{n}$, and $\theta_{n} c$ is the access cost. Define the ratio between the utility and active probability of user $n$ as $v_{n}=u_{n} / \theta_{n}$, which successfully reflects the efficiency of transmitting data. We use Jain's Fairness Index (JFI) [13] to describe system fairness as

$$
V_{j f i}=\frac{\left(\sum_{n=1}^{N} v_{n}\right)^{2}}{N \sum_{n=1}^{N} v_{n}^{2}} .
$$

The system objective in this paper is to find the optimal channel selection profile $\vec{a}_{\text {opt }}=\left(a_{1}, a_{2}, \ldots, a_{N}\right)$ to maximize each user's rewards $u_{i}$. Formally,

$$
\vec{a}_{\text {opt }}=\arg \max u_{i}, \forall i \in \mathbf{N} .
$$

It may be seen that solving the problem in Eq. (6) is a challenge, since (i) there is no control center for user to coordinate, and (ii) users do not know other users' strategies, actions and active probabilities. Thus, a distributed approach for users' dynamic participation in an unknown dynamic environment is desirable. 


\section{Directional Graphical Game and Stochastic Learning Approach}

\subsection{Directional Graphical Game Model with Dynamic Users}

We formulate the channel selection problem as a non-cooperative game because the users make their own decisions in a distributive and autonomous manner. The game is denoted as $\mathbf{G}=\left\{\mathbf{N}, \mathbf{A}_{n}, \mathbf{J}_{n}, u_{n}\right\}$, where $\mathbf{N}$ is the users set and $\mathbf{A}_{n}$ is the action space of the user $n, \mathbf{J}_{n}$ is the topology of the user $n$ and $u n$ is the utility function. The action space of all users is exactly the channel set, i.e., $\mathbf{A}_{n}=M$, and the topology, $\mathbf{J}_{n}$, of user $n$ is the user's neighbor set. Generally, the utility function is denoted as $u_{n}\left(a_{n}, a_{-n}\right)$, where $a_{n}$ is the action of the user $n$ and $a_{-n}$ is the action set of other users excluding the user $n$. According to the graphical model, the user $n$ can only be affected by its neighbors instead of all other users. Thus, the utility function may be expressed as $u_{n}\left(a_{n}, a_{\mathbf{J}_{n}}\right)$, where $a_{\mathbf{J}_{n}}$ is the action set of user's neighbors. We define the utility function as

$$
u_{n}\left(a_{n}, a_{\mathbf{J}_{n}}\right)=R_{a_{n}} \theta_{n} \prod_{i \in \mathbf{J}_{n}, a_{i}=a_{n}}\left(1-\theta_{i}\right) R_{a_{n}}-\theta_{n} c
$$

The utility function is the same as the throughput reward considering access cost of user $n$. Then, the proposed channel selection game with users' dynamic participation is expressed as

$$
\mathbf{G}: \max u_{n}\left(a_{n}, a_{\mathbf{J}_{n}}\right), \forall n \in \mathbf{N}
$$

\subsection{Analysis of the Nash Equilibrium}

In this subsection, we first define the Nash equilibrium (NE) and the exact potential game of the game $\mathbf{G}$.

Definition 1 (Nash Equilibrium): A channel selection profile $a_{N E}=\left(a_{1}^{*}, a_{2}^{*}, \ldots, a_{N}^{*}\right)$ is a pure strategy $\mathrm{NE}$ if and only if no user can improve its utility by deviating unilaterally, i.e.,

$$
u_{n}\left(a_{n}^{*}, a_{\mathbf{J}_{n}}^{*}\right) \geq u_{n}\left(\bar{a}_{n}, a_{\mathbf{J}_{n}}^{*}\right), \forall n \in \mathbf{N}, \forall \bar{a}_{n} \in \mathbf{A}_{n}, \bar{a}_{n} \neq a_{n}^{*}
$$

Definition 2 (Exact Potential Game) [14]: A game $\mathbf{G}=\left[\mathbf{N}, \mathbf{A}_{n}, \mathbf{J}_{n}, u_{n}\right]$ is an exact potential game if there exists a function $\Phi$ such that

$$
\Phi\left(\bar{a}_{n}, a_{-n}\right)-\Phi\left(a_{n}, a_{-n}\right)=u_{n}\left(\bar{a}_{n}, a_{\mathbf{J}_{n}}\right)-u_{n}\left(a_{n}, a_{\mathbf{J}_{n}}\right), \forall \bar{a}_{n} \in \mathbf{A}_{n}, \bar{a}_{n} \neq a_{n}^{*}
$$

The function $\Phi$ is called an exact potential function for the game $\mathbf{G}$.

Theorem 1: The channel selection game $\mathbf{G}$ is an exact potential game which has at least one pure strategy NE point.

Proof: To prove this theorem, we form a potential function as

$$
\Phi\left(a_{n}, a_{-n}\right)=\sum_{m \in M}\left[1-\prod_{i \in \in \mathbf{J}_{n}+n, a_{i}=m}\left(1-\theta_{i}\right)\right] R_{m},
$$

where $\sum_{m \in M}\left[1-\prod_{i \in \in \mathbf{J}_{n}+n, a_{i}=m}\left(1-\theta_{i}\right)\right]$ is the idle probability of channel $m$. The potential function's physical meaning is the sum occupancy of resource for user $n$.

Suppose that an arbitrary player $n$ unilaterally changes its channel selection from $a_{n}$ to $\bar{a}_{n}$. Then, the change of the utility function reads 


$$
\begin{aligned}
& u_{n}\left(\bar{a}_{n}, a_{\mathbf{J}_{n}}\right)-u_{n}\left(a_{n}, a_{\mathbf{J}_{n}}\right) \\
& =R_{\bar{a}_{n}} \theta_{n} \prod_{a_{i}=\bar{a}_{n}}\left(1-\theta_{i}\right)-\theta_{n} c-R_{a_{n}} \theta_{n} \prod_{a_{i}=a_{n}}\left(1-\theta_{i}\right)+\theta_{n} c \\
& =R_{\bar{a}_{n}} \theta_{n} \prod_{a_{i}=\bar{a}_{n}}\left(1-\theta_{i}\right)-R_{a_{n}} \theta_{n} \prod_{a_{i}=a_{n}}\left(1-\theta_{i}\right)
\end{aligned}
$$

When user $n$ changes its channel selection from $a_{n}$ to $\bar{a}_{n}$, it only affects these two channels occupy situations and other channels are unaltered. Thus, the change of the potential function is made up of only two occupy situations of channel $a_{n}$ and $\bar{a}_{n}$. Formally,

$$
\begin{aligned}
& \Phi\left(\bar{a}_{n}, a_{-n}\right)-\Phi\left(a_{n}, a_{-n}\right) \\
& =R_{a_{n}}\left[1-\prod_{a_{i}=a_{n}}\left(1-\theta_{i}\right)\right]+R_{\bar{a}_{n}}\left[1-\left(1-\theta_{n}\right) \prod_{a_{i}=\bar{a}_{n}}\left(1-\theta_{i}\right)\right] \\
& -R_{a_{n}}\left[1-\left(1-\theta_{n}\right) \prod_{a_{i}=a_{n}}\left(1-\theta_{i}\right)\right]-R_{\bar{a}_{n}}\left[1-\prod_{a_{i}=\bar{a}_{n}}\left(1-\theta_{i}\right)\right] \\
& =R_{\bar{a}_{n}} \theta_{n} \prod_{a_{i}=\bar{a}_{n}}\left(1-\theta_{i}\right)-R_{a_{n}} \theta_{n} \prod_{a_{i}=a_{n}}\left(1-\theta_{i}\right) \\
& =u_{n}\left(\bar{a}_{n}, a_{\mathbf{J}_{n}}\right)-u_{n}\left(a_{n}, a_{\mathbf{J}_{n}}\right)
\end{aligned}
$$

It may be seen from Eq. (13) that the change of the utility function, caused by any player's unilateral deviation, is the same as the change of the potential function. According to the definition of the exact potential game [14], the channel selection game $\mathbf{G}$ is an exact potential game with potential function $\Phi$ and there is at least one pure strategy NE point. Therefore, Theorem 1 is proved.

Remark 2: Although higher resource occupancy does not always lead to higher throughput, it is still a necessary condition to achieve a high throughput. The NE point of the directional graphical game $\mathbf{G}$ maximizes the resource occupancy and the utility of each user with a large probability at the same time. Hence, it is important and necessary to find the NE point of the game.

\subsection{Modified Stochastic Learning Approach}

\section{Algorithm 1: Modified stochastic learning approach}

Initialization: In $j=0$ slot, each user initializes the channel selection probability vector $p_{n m}(j)=1 / M, \forall n \in \mathbf{N}, m \in\{1, \ldots, M\}$.

Loop for: $j=1,2, \ldots$,

At the beginning of the $j$ th slot, each user decides to be active or not according to its active probability. For the active users, they select one channel based on their channel selection probability $\mathbf{p}_{n}(j)$. Users successfully transmit their data or collide with other users. At the ending of $j$ th slot, each user $n$ receives a rewards $r_{n}(j)$ specified by Eq. (3). All the users compute a temporary channel selection probability by the following rules:

$$
\begin{aligned}
& p_{n m}^{\prime}(j+1)=p_{n m}(j)+b r_{n}(j)\left(1-p_{n m}(j)\right), m=a_{n}(j) \\
& p_{n m}^{\prime}(j+1)=p_{n m}(j)-b r_{n}(j) p_{n m}(j), m \neq a_{n}(j)
\end{aligned}
$$


where $0<b<1$ is the learning parameter. Update the channel selection probabilities by Eq. (15):

$$
p_{n m}(j+1)=\left\{\begin{array}{l}
p_{n m}(j), \exists m \in \mathbf{M}, p_{n m}^{\prime}(j+1)<0, \\
p_{n m}^{\prime}(j+1), \text { otherwise }
\end{array}\right.
$$

which means if the temporary probability is not possible, i.e. $p_{n m}^{\prime}(j+1)<0$, the channel selection probability keeps the same, and if possible, it updates as the temporary probabilities. End loop

To achieve the NE point of the potential games, many algorithms have been reported, such as the best response [15], fictitious play [16], and spatial adaptive play [8]. However, some of them require to know all other users' actions of the game, which is hard to achieve in the distributed systems. In this paper, we use the stochastic learning approach [5] to achieve the NE point of the channel selection game, which is described in Algorithm 1.

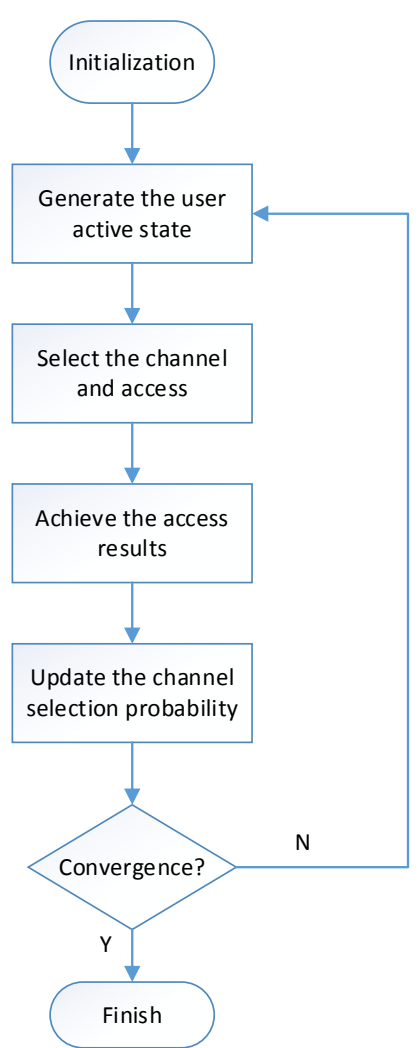

Fig. 2. The flow chart of the modified stochastic learning algorithm.

We slightly adjust the algorithm to adapt it to the dynamic users. In the proposed system model, users are not always learning. In fact they are inactive sometimes. Thus, when users are active, they update their channel selection probabilities according to the updating rules, and when inactive, the channel selection probabilities keep the same. When users fail to access the channel, the utility function of the users may be negative. Based on the design of the updating rules, the mechanism of negative rewards decreases the selection probability of the failed 
channel but increases the probabilities of other channels. Through the negative utility, users learn from the failure and update their strategies. When the channel selection probability $p_{n m}$ is close to zero, it may become negative if it is punished again. To avoid this situation, the temporary probabilities are checked before updating. In this way, the channel selection probabilities keep in the range from zero to one. The flow chart of the modified SLA is shown in Fig. 2.

Theorem 2. The stochastic learning approach based on the dynamic users converges to a pure NE point of $G$.

Proof: The proof is similar with the Theorem 6 in [5] and is omitted here.

Remark 3: It is noted that the SLA algorithm finally converges to the NE point, which maximizes the personal utility and a large possible to maximize system utility. The three kinds of rewards, i.e., positive, negative and zero, match the three kinds of user behaviors, i.e., successful, failed or inactive. Compared with another SLA algorithm [5], which has only positive and zero rewards, the negative rewards make users adjust their strategies through the failed experience, similar with the negative feedback. The designed updating rules ensure that the channel selection probability has a clear physical meaning.

\section{Simulation Results and Discussion}

This Section presents the simulation results of the stochastic learning approach (SLA) algorithm. First, the convergence behavior of the algorithm is given out. Then, we compare the algorithm's throughput and fairness performance with the exhaustive approach algorithm and random access. All the users are located in a $1000 \mathrm{~m} \times 1000 \mathrm{~m}$ square area. The interference range set of the users is $[250 ; 300 ; 350]$ and the users randomly select one from the set as their range in the simulation. The channel rate is normalized, $0<R_{m}<1, m \in \mathbf{M}$. The presented results are achieved by simulating 5000 independent trials, where each trial relocates the users position, and reset the active probabilities and the channel rates.

\subsection{Convergence Behavior}

Fig. 3 shows the channel selection probability convergence. Consider an arbitrary user of the OSA system with three channels and ten users. The learning algorithm updates the probabilities. Finally, the probability of the selection of Channel 2 increases nearly to one and the probabilities of the selection of other channels decrease to zero. When the iteration is about 110 , it is shown that these probabilities remain the same for several continuous slots. This is due to the fact that users are inactive in these slots and they do not learn and update.

Fig. 4 shows the modified SLA convergence cumulative distribution function (CDF). We change the active probability from 0.3 to 0.7 with 10 users. From the figure, with the active probability increasing, the algofithm convergences more rapidly. According to the simulation results, the average convergence iteration is about 418, 299 and 236 for the corresponding probability. 


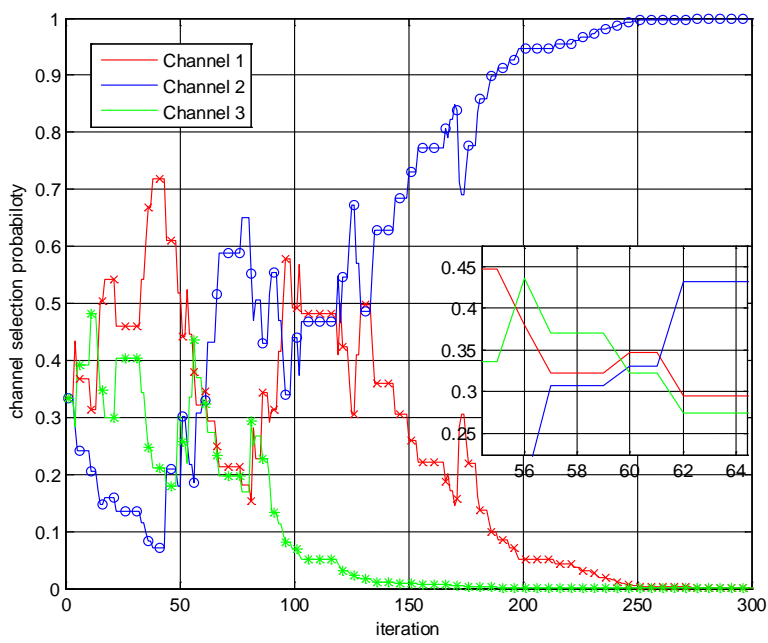

Fig. 3. Channel selection probabilities evolutionary process of a user.

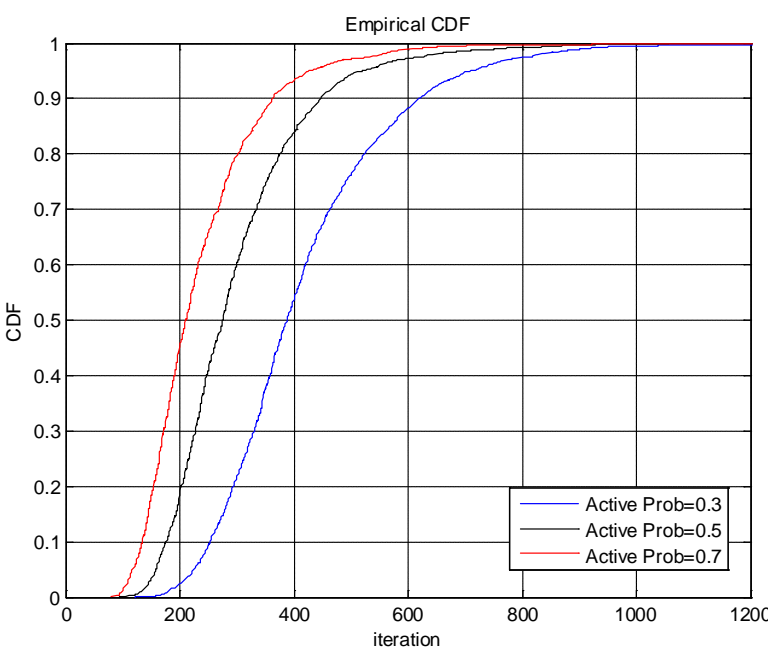

Fig. 4. The modified SLA convergence CDF with different active probabilities.

\subsection{Throughput and Fairness Performance}

Then we compare the SLA algorithm with other algorithms, i.e. the exhaustive approach and a random access algorithm. A center controller in the exhaustive approach searches all the channel selection strategies and finds the optimal one as the final selection, while the random approach randomly selects the channels.

Fig. 5 plots the utility performance. Since the exhaustive approach searches the best channel selection from all the possible ones, too much time is required for a large number of users. Thus, we only simulated the exhaustive approach when number of users $N$ is less than 10. The SLA algorithm provides better performance than the random approach and is close to the exhaustive approach. Fig. 6 compares the fairness of the algorithms. The JFI of SLA algorithm is above 0.9 , which represents a good fairness. 


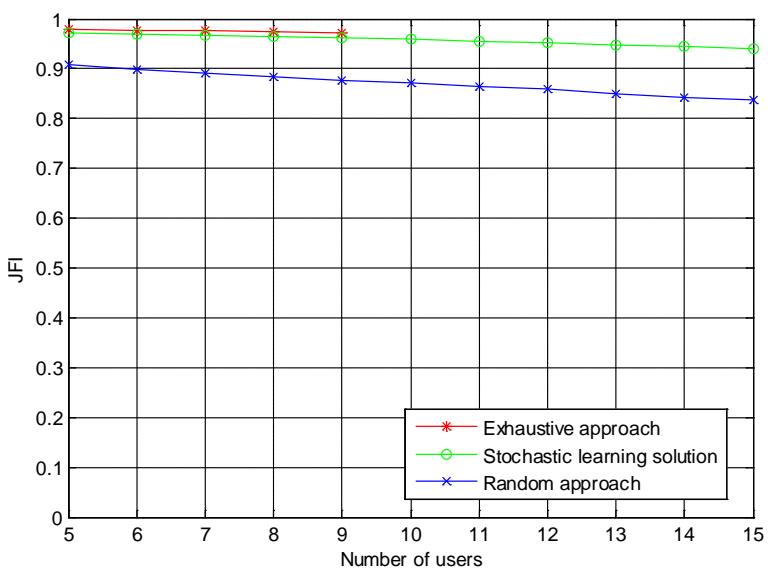

Fig. 5. Utility performance comparison.

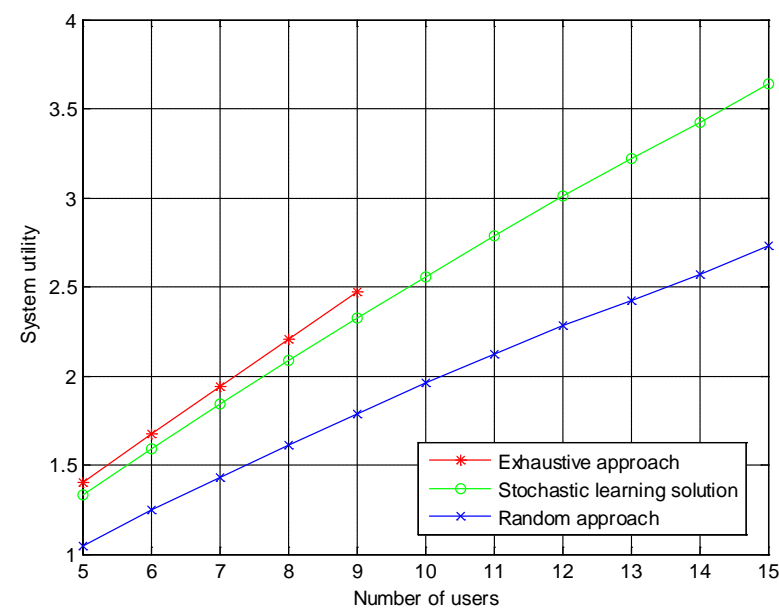

Fig. 6. Fairness performance comparison.

Fig. 7 investigates the throughput with different number of users and active probabilities. With the increase of the number of users $N$ and active probabilities $\theta$, the system utility initially increases but finally decreases after $N$ reaches a large number and $\theta$ close to one. The reason is that for too many active users in the system, each user is affected by too many other users and the system congestion significantly increases to an intolerant level. As a result of the high collusion, the system utility decreases. Based on the ALOHA mechanism in the system model, for two or more active users in a channel, neither of them would successfully accesses and receives a reward. Therefore, when the numbers and active probabilities of the users are increasing up to some certain value, the users cannot avoid the contention anymore and the network throughput slowly increases and even decreases, just as the curve of $\mathrm{N}=30$. 


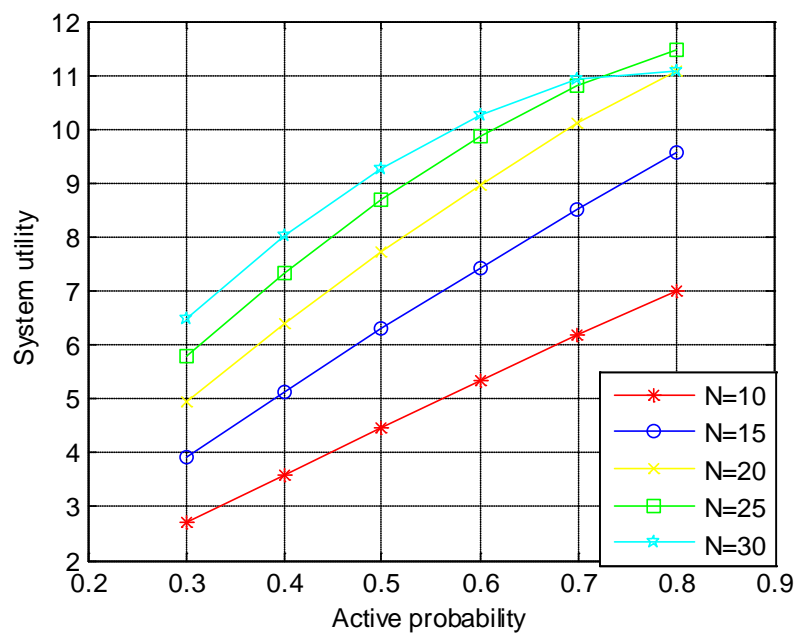

Fig. 7. Utility comparison in the homogeneous systems.

\subsection{Throughput in Different Asymmetric Level Systems}

Fig. 8 illustrates the classification of the interference asymmetric levels into three types, i.e. all-asymmetric, partly-asymmetric and symmetric levels. We also compare the throughput performance in different interference asymmetric level systems. As Fig. 9 plots, the asymmetric situations are better than the symmetric ones. About $15 \%$ and $24 \%$ improvement compared with symmetric situation for 25 users, while the advantage is increasing with the number of users. Therefore, the directional graphical model is a more exact description and significantly promotes the systems throughput.

Above all, the simulation results indicate that: (i) The SLA algorithm converges well for all types of system models. (ii) The SLA algorithm gives good utility close to exhaustive approach. (iii) The fairness performance of the SLA algorithm is also good, and (iv) compared with non-directional model, the directional promotes the throughput performance.

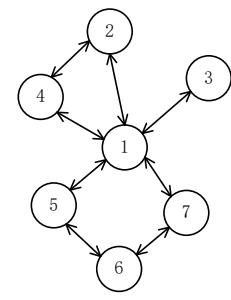

Symmetric

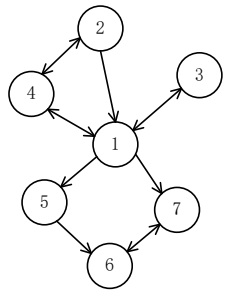

Part-asymmetric

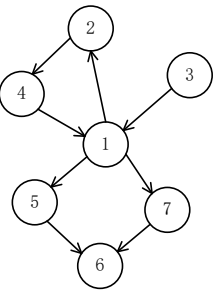

All-asymmetric

Fig. 8. Different asymmetric level models. 


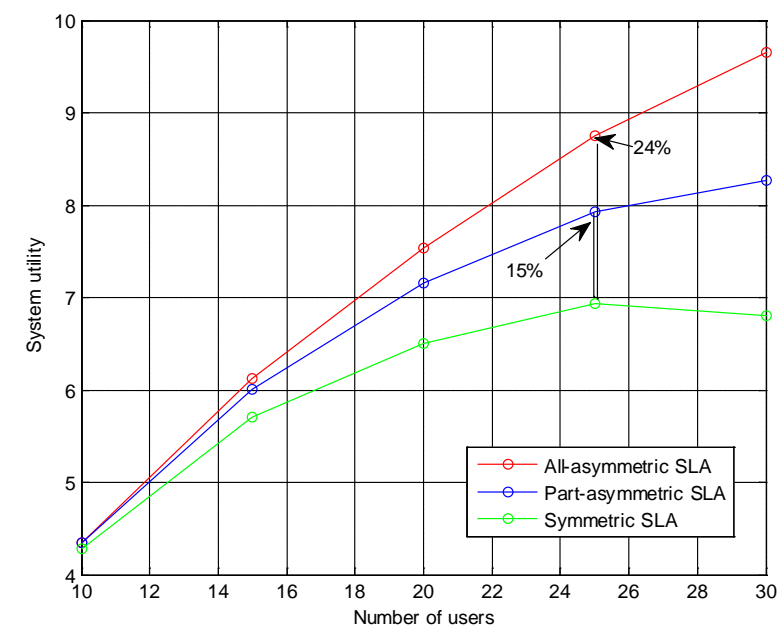

Fig. 9. Utility comparison in different interference asymmetric level systems.

\section{Conclusion}

The channel selection problem in the distributed OSA systems with dynamic users is studied in this paper. To show the users dynamic characteristics, we assumed that at each time slot, users randomly transmit the data. To address the asymmetric interference relation between the users, the problem is modeled into a directional graphical game. We proved the directional graphical game as an exact potential game, having at least one NE point. It is also shown that the best NE point maximizes the personal utility with the system utility. The stochastic learning algorithm is modified for the system. The simulation results verified the convergence of the stochastic learning algorithm, achieving near-optimal performance with good fairness. The throughput in different asymmetric level systems is compared and found the directional graphical model that improves the systems utility.

\section{Acknowledgement}

This work was supported in part by the Natural Science Foundation for Distinguished Young Scholars of Jiangsu Province under Grant BK20160034, in part by the National Science Foundation of China under Grant 61631020, Grant 61671473, and Grant 61401508, and in part by the Open Research Foundation of Science and Technology on Communication Networks Laboratory.

\section{References}

[1] Y. Xu, A. Alagan and Q. Wu et al., "Decision-theoretic distributed channel selection for opportunistic spectrum access: Strategies, challenges and solutions," IEEE Commun. Survey \& Tutorials, vol. 15, no. 4, pp. 1689- 1713, 2013. Article (CrossRef Link)

[2] Q. Zhao and B. M.Sadler, "A survey of dynamic spectrum access," IEEE Signal Process. Magazine, vol. 24. no. 3, pp. 79-89, 2007. Article (CrossRef Link)

[3] H. Li and Z. Han, "Competitive spectrum access in cognitive radio networks: Graphical game and learning,” in Proc. of 2010 IEEE WCNC, pp. 1-6, 2010. Article (CrossRef Link) 
[4] Y. Xu, Q. Wu, L. Shen, et al., "Opportunistic spectrum access with spatial reuse: Graphical game and uncoupled learning solution,” IEEE Trans. Wireless Commun., vol.12, no. 10, pp. 4814-4826, 2013. Article (CrossRef Link)

[5] Y. Xu, J. Wang, Q. Wu, et al,. “Opportunistic spectrum access in unknown dynamic environment: A game-theoretic stochastic learning solution,” IEEE Trans. Wireless Commun., vol. 11, no.4, pp.1380-1391, 2012. Article (CrossRef Link)

[6] M. Azarafrooz and R. Chandramouli, "Distributed learning in secondary spectrum sharing graphical game,” in Proc. of 2011 IEEE GLOBECOM, pp. 1-6, 2011. Article (CrossRef Link)

[7] M. Liu, S. Ahmad, and Y.Wu, "Congestion games with resource reuse and applications in spectrum sharing” GameNets, pp. 171-179, 2009. Article (CrossRef Link)

[8] Y. Xu, J. Wang, Q. Wu, et al, “Opportunistic spectrum access in cognitive radio networks: Global optimization using local interaction game,” IEEE J. Sel. Topics Signal Process., vol. 6, no. 2, pp. 180-194, 2012. Article (CrossRef Link)

[9] Y. Xu, Q. Wu, J. Wang, et al., "Opportunistic spectrum access using partially overlapping channels: Graphic game and uncoupled learning," IEEE Trans. Commun., vol. 61, no. 9, pp. 3906-3918, 2013. Article (CrossRef Link)

[10] J. Zheng, Y. Cai, Y. Xu, and A. Anpalagan, "Distributed channel selection for interference mitigation in dynamic environment: A game-theoretic stochastic learning solution,” IEEE Trans. Vehicular Technology, vol. 63, no. 9, pp. 4757-4762, 2014. Article (CrossRef Link)

[11] X. Chen, and J. Huang, "Distributed spectrum access with spatial reuse," IEEE J. Sel. Areas Commun., vol. 31, no. 3, pp. 593-603, 2013. Article (CrossRef Link)

[12] IEEE 802.16e-2005 and IEEE Std 802.16-2004/Corl-2005. Article (CrossRef Link).

[13] R. Jain, D. Chiu, and W. Haws, “A quantitative measure of fairness and discrimination for resource allocation in shared computer system,” Technical Report, 1984. Article (CrossRef Link)

[14] D. Monderer and L. S. Shapley, "Potential games," Game Economic Behacior, vol. 14, pp. 124-143, 1996. Article (CrossRef Link)

[15] P. Duarte, Z. Fadlillah, A. Vasilakos and N. Kato, "On the partially overlapped channel assignment on wireless mesh network backone: A game theoretic approach,” IEEE J. Sel. Areas Commun., vol. 30, no. 1, pp. 119- 127, 2012. Article (CrossRef Link)

[16] J. Marden, G. Arslan and J. Shamma, "Joint strategy fictitious play with inertia for potential games,” IEEE Trans. Autom. Control, vol. 54, no. 2, pp. 208-220, 2009. Article (CrossRef Link)

[17] Q. Wu, D. Wu, Y. Xu and J. Wang, "Demand-Aware Multichannel Opportunistic Spectrum Access: A Local Interaction Game Approach With Reduced Information Exchange,” IEEE Transactions on Vehicular Technology, vol. 64, no. 10, pp. 4899-4904, Oct. 2015. Article (CrossRef Link)

[18] L. Xiao, H. Dai and P. Ning, "Jamming-Resistant Collaborative Broadcast Using Uncoordinated Frequency Hopping,” IEEE Trans. Information Forensics \& Security, vol. 7, no. 1, pp. 297 - 309, Feb. 2012. Article (CrossRef Link)

[19] N. Hasan and et al., "Network Selection and Channel Allocation for Spectrum Sharing in 5G Heterogeneous Networks,” IEEE Access, vol. 4, pp. 980-992, March 2016. Article (CrossRef Link)

[20] Darsena D., Gelli, G. and Verde, F., “An Opportunistic Spectrum Access Scheme for Multicarrier Cognitive Sensor Networks,” IEEE Sensors Journal, vol.17, no.8, pp.2596-2606, 2017. Article (CrossRef Link)

[21] Akhtar, F., Rehmani, M.H. and Reisslein, M., "White space: Definitional perspectives and their role in exploiting spectrum opportunities,” Telecommunications Policy, vol.40, no.4, pp.319-331, 2016. Article (CrossRef Link)

[22] Pratibha, P., Li, K.H. and Teh, K.C., "Optimal Spectrum Access and Energy Supply for Cognitive Radio Systems with Opportunistic RF Energy Harvesting,” IEEE Transactions on Vehicular Technology, Vol.66, Issue 8, pp.7114-7122, 2017. Article (CrossRef Link)

[23] Khan, A.A., Rehmani, M.H. and Reisslein, M., "Cognitive radio for smart grids: Survey of architectures, spectrum sensing mechanisms, and networking protocols," IEEE Communications Surveys \& Tutorials, 18(1), pp.860-898, 2016. Article (CrossRef Link) 
[24] X. Zhang, N. Zhao, F. Richard Yu, Minglu Jin, and Victor C. M. Leung, "Resource Allocation in Topology Management of Asymmetric Interference Networks” IEEE Systems Journal, Issue 99, pp.1-11, 2017. Article (CrossRef Link)

[25] N. Zhao, X. Zhang, F. Richard Yu, Victor Leung, "To Align or Not to Align: Topology Management in Asymmetric Interference Networks," IEEE Transactions on Vehicular Technology, Vol.66, Issue 8, pp. 7164-7177, 2017. Article (CrossRef Link)

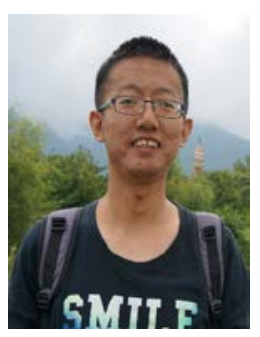

Yuli Zhang received his B.S. degree in electronic and information engineering from Peking University, Beijing, China, in 2012 and M.S. degree from College of Communications Engineering, PLA University of Science and Technology, Nanjing, China, in 2015, respectively. He is currently a Ph.D. candidate in College of Communications Engineering, PLA University of Science and Technology. His research interests include resource allocation in small cell networks, game theory and spectrum market.

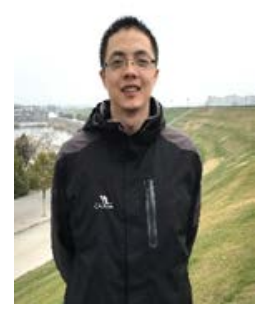

Yuhua Xu received his B.S. degree in Communications Engineering, and Ph.D. degree in Communications and Information Systems from College of Communications Engineering, PLA University of Science and Technology, in 2006 and 2014 respectively. He has been with College of Communications Engineering, PLA University of Science and Technology since 2012, and currently as an Assistant Professor. His research interests focus on opportunistic spectrum access, learning theory, game theory, and distributed optimization techniques for wireless communications. He has published several papers in international conferences and reputed journals in his research area.

He served as Associate Editor for Wiley Transactions on Emerging Telecommunications Technologies and KSII Transactions on Internet and Information Systems, and the Guest Editor of Special Issue on "The Evolution and the Revolution of 5G Wireless Communication Systems” for IET Communications. In 2011 and 2012, he was awarded Certificate of Appreciation as Exemplary Reviewer for the IEEE Communications Letters. He was selected to receive the IEEE Signal Processing Society's (SPS) 2015 Young Author Best Paper Award, and the Funds for Distinguished Young Scholars of Jiangsu Province in 2015.

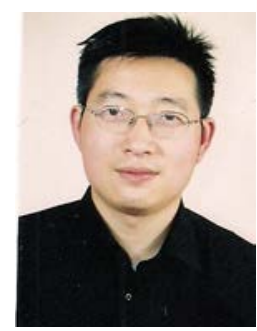

Qihui Wu received the B.S. degree in communications engineering and the M.S and Ph.D. degrees in communications and information system from the PLA University of Science and Technology, Nanjing, China, in 1994, 1997, and 2000, respectively. He is currently a Professor with the College of Electronic and Information Engineering, Nanjing University of Aeronautics and Astronautics. His current research interests include algorithms and optimization for cognitive wireless networks, soft-defined radio, and wireless communication systems. 